\title{
INFLAMMATORY PROFILE IN OLDER ADULTS WITH HEIGHTENED TRAJECTORIES OF DEPRESSION SYMPTOMS
}

\section{A. de La Torre-Luque, Y. Sanchez-Carro, J. De la Fuente, P. Lopez-Garcia, J.L. Ayuso-Mateos.}

Centre for Biomedical Research in Mental Health CIBERSAM, Madrid, Spain. Universidad Autonoma de Madrid, Spain.

Contact: joseluis.ayuso@uam.es

\section{BACKGROUND AND OBJECTIVES}

- Depression may be linked with some devastating effects in old age (e.g., increased mortality risk, higher disease burden). Some studies have shown prevalence rates of late-life depression of 30-40\% [1,2]. Alterations in pro-inflammatory activity (e.g., increased cytokine release) and low-grade inflammation have been well documented in individuals with high levels of depressive symptoms [3-6]. However, longitudinal studies are needed to explain how depressive symptom course may be connected with specific inflammation profiles while ageing.

- This study aimed to examine the relationships between inflammatory profiles and depression symptom trajectories while ageing.

\section{METHOD}

Data from 1,536 participants aged 50-90 years (56.58\% women; mean age at baseline $=61.73$ years, $s d=7.64$ ) from the English Longitudinal

Figure 1. Depressive symptom trajectories.

Study of Ageing (ELSA) were used.

Longitudinal trajectories of depressive symptoms (CES-D 8 scores) were identified (Figure 1) over a 10-year follow-up (2002-2012), using latent class mixed modelling.

Inflammation profile scores were estimated from plasma indicators (C-reactive protein, fibrinogen, white blood cell count). Sex, age, history of emotional problems, antidepressant prescription and multimorbidity were used as covariates. Blood samples were drawn in 2008.

Multigroup latent-variable modelling was used to study the

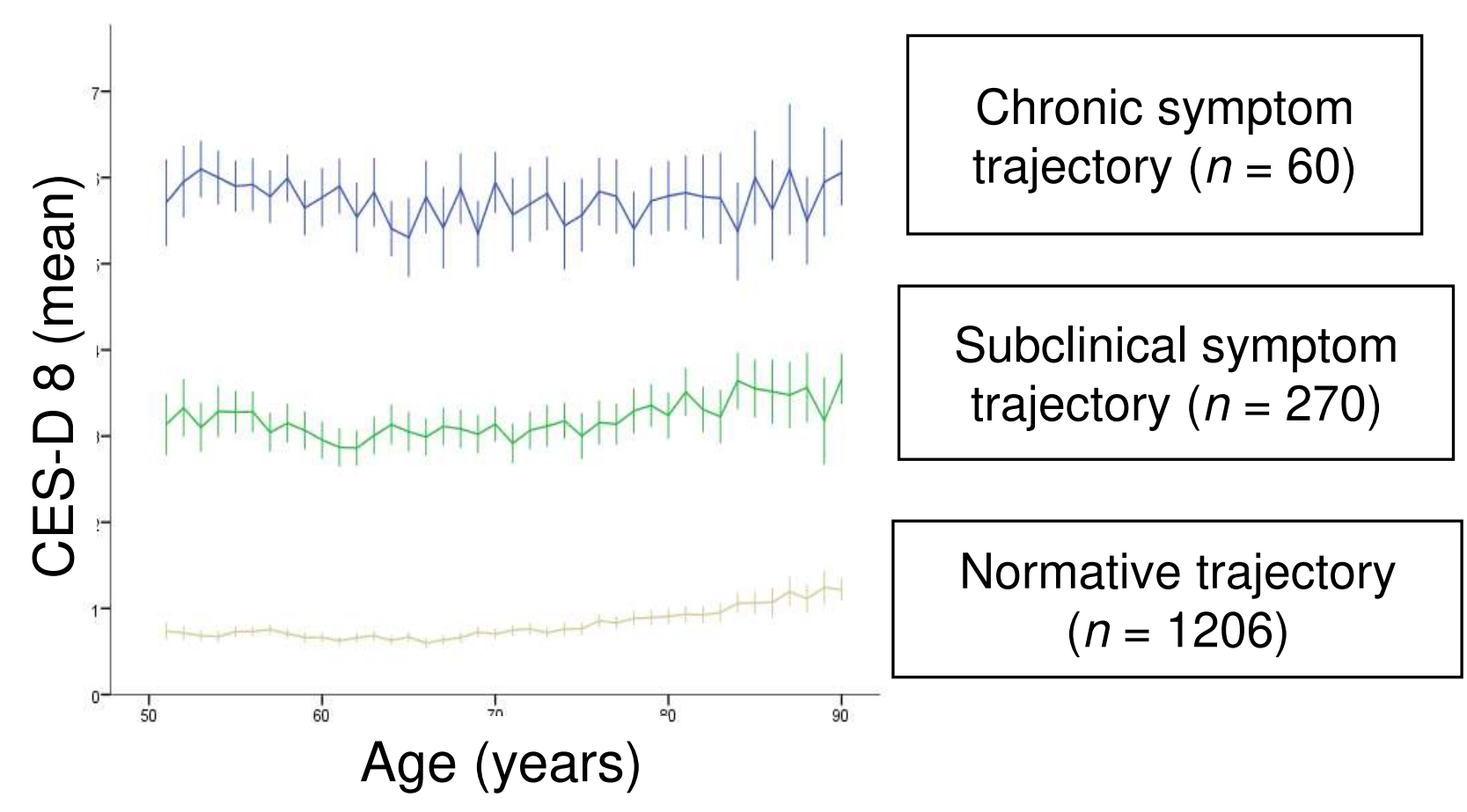
relationships between this profile and symptom trajectories.

\section{RESULTS}

Figure 2. Inflammation indicators and score across trajectory classes.
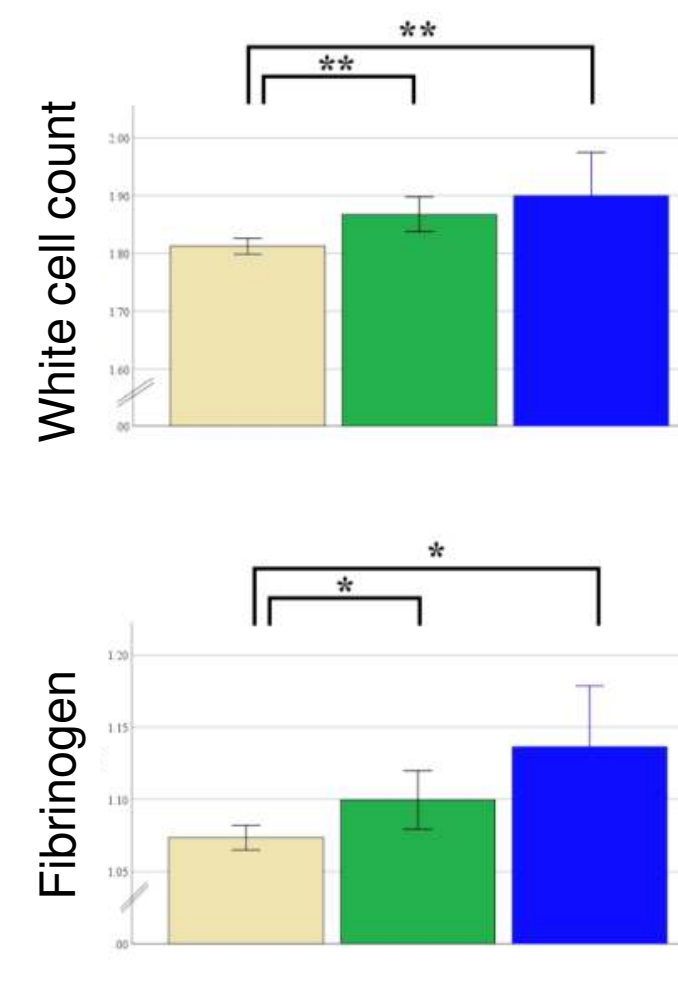

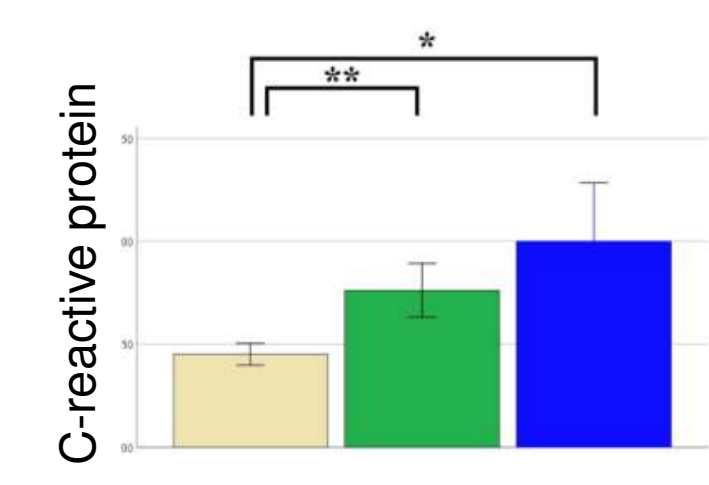

$\square$ Normative trajectory
$\square$ Subclinical symptom trajectory
$\square$ Chronic symptom trajectory

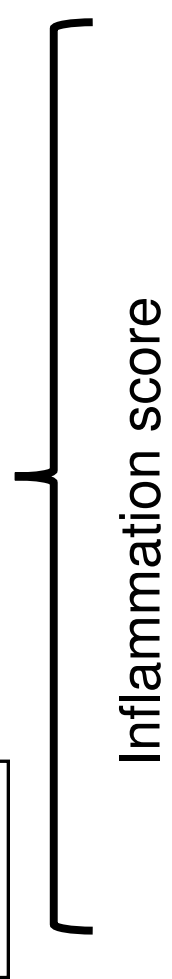

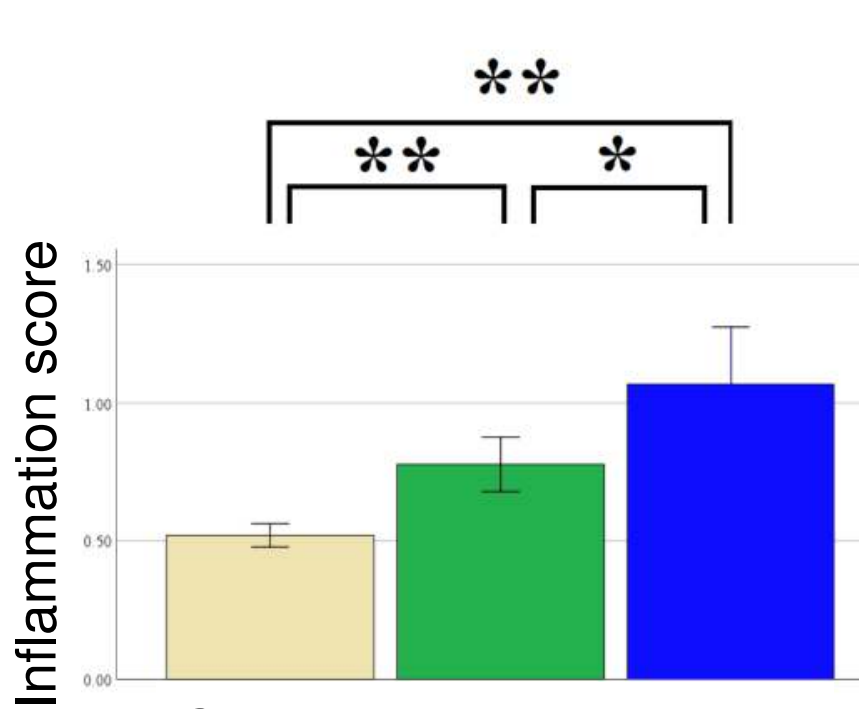

Symptom trajectory class

${ }^{*} p<.05 ;{ }^{* *} p<.01$
Multigroup analysis revealed a non-significant loss of fit of the inflammation profile model when indicator loadings, intercepts and residuals were constrained across symptom trajectory classes, $\triangle \mathrm{CFI}$ $=.004, \triangle \mathrm{RMSEA}=-.005$.

Significant differences across classes were shown for all indicators and inflammation score (Figure 2) The more severe (heightened) the symptom trajectories the higher the levels of inflammatory biomarkers and latent factor score.

\section{CONCLUSIONS}

Heightened trajectories of depression symptoms were associated with higher inflammation profiles, pointing to the peripheral inflammatory profile as a potential biomarker of elevated symptom courses. Dysregulation was also observed in the subclinical symptom trajectory. These results are in line with integrating longitudinal and person-centred corollaries to personalised medicine and mental health precision. 